INPLASY

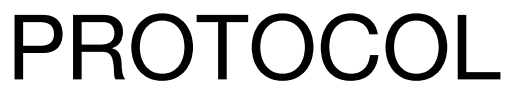

To cite: Ge et al. Identification of the functional and predictive roles of MAGEA4 in taking nonsteroidal anti-inflammatory drugs during pregnancy increases the risk of cryptorchidism. Inplasy protocol 202070076. doi: 10.37766/inplasy2020.7.0076

Received: 16 July 2020

Published: 16 July 2020

Corresponding author:

Yunzhao Xu

xuyz@ntu.edu.cn

Author Affiliation:

Affiliated Hospital of Nantong University

Support: None.

Review Stage at time of this submission: The review has not yet started.

Conflicts of interest:

None.

\section{Identification of the functional and predictive roles of MAGEA4 in taking nonsteroidal anti-inflammatory drugs during pregnancy increases the risk of cryptorchidism}

Ge, WL1; Ren, SQ2; Xu, YZ3 .

Review question / Objective: As we all know, almost $10 \%$ of testicular tumors are caused by cryptorchidism. In the development of germ cell tumors, the most dangerous factor is cryptorchidism. During pregnancy, the use of non-steroidal anti-inflammatory drugs is common. However, research on the adverse effects of NSAIDs on neurodevelopmental outcomes in prenatal children is still lacking. Recently there have been reports that prolonged use (more than 4 weeks) of paracetamol during pregnancy increases the risk of cryptorchidism. Therefore, we conducted a systematic review and meta-analysis to determine the relationship between cryptorchidism and the use of NSAIDs during pregnancy.

INPLASY registration number: This protocol was registered with the International Platform of Registered Systematic Review and Meta-Analysis Protocols (INPLASY) on 16 July 2020 and was last updated on 16 July 2020 (registration number INPLASY202070076).

\section{INTRODUCTION}

Review question / Objective: As we all know, almost $10 \%$ of testicular tumors are caused by cryptorchidism. In the development of germ cell tumors, the most dangerous factor is cryptorchidism. During pregnancy, the use of non-steroidal anti- inflammatory drugs is common. However, research on the adverse effects of NSAIDs on neurodevelopmental outcomes in prenatal children is still lacking. Recently there have been reports that prolonged use (more than 4 weeks) of paracetamol during pregnancy increases the risk of cryptorchidism. Therefore, we conducted a 
systematic review and meta-analysis to determine the relationship between cryptorchidism and the use of NSAIDs during pregnancy.

Condition being studied: As we all know, almost $10 \%$ of testicular tumors are caused by cryptorchidism. In the development of germ cell tumors, the most dangerous factor is cryptorchidism. Medication is very common during pregnancy. The occurrence of pre-eclampsia during high-risk pregnancy can be controlled by using lowdose NSAIDs before 16 weeks of pregnancy. Nevertheless, the use of nonsteroidal anti-inflammatory drugs is common during pregnancy. However, research on the adverse effects of NSAIDs on neurodevelopmental outcomes in prenatal children is still lacking. Recently there have been reports that prolonged use (more than 4 weeks) of paracetamol during pregnancy increases the risk of cryptorchidism.

\section{METHODS}

Search strategy: (NSAIDs OR analges) AND (Cryptorchidism OR undescended test OR non-descended test OR non descended test).

Participant or population: 1408 pregnant women and 780 children with cryptorchidism.

Intervention: Use of NSAIDs during pregnancy.

\section{Comparator: None.}

Study designs to be included: One arm study, randomized controlled trial.

Eligibility criteria: The papers included in this study meets the following inclusion criteria: (i) mothers of children with cryptorchidism have a history of exposure to an.

Information sources: Pubmed, Embase and Cochrane library.
Main outcome(s): We calculated the effect size (ES) and $95 \%$ confidence interval (CI) of cryptorchidism in each study.

Data management: Shiqi Ren and Wenliang Ge independently extracted the data needed for single arm meta-analysis in the included study, including the author's name, study year, follow-up time, experimental type, number of cryptorchidism cases, number of cryptorchidism cases exposed to analgesics, type of analgesics and outcome measurement method. The difference between the two authors was referred to the third reviewer (Yunzhao Xu).

Quality assessment / Risk of bias analysis: We used the Cochrane risk of bias assessment to assess the bias risk of the included research. Besides the other sources of bias, selective outcome reporting, incomplete outcome data, blinding of outcome assessment, personnel, blinding of participants, allocation concealment, and randomized Sequence Generation are included in the evaluation. Shiqi Ren and Wenliang Ge completed the evaluation of each document independently. The difference between the two authors was referred to other authors.

Strategy of data synthesis: We calculated the effect size (ES) and $95 \%$ confidence interval $(\mathrm{Cl})$ of cryptorchidism in each study. $Q$ test and 12 statistical methods were used to evaluate the statistical heterogeneity of the study. The $P<0.05$ of $Q$ test showed that there was significant heterogeneity, and $12>50 \%$ was considered as statistically significant heterogeneity. If the $12>50 \%$, we used the random effect model for single arm metaanalysis.

Subgroup analysis: We conducted a subgroup analysis to investigate whether the use of analgesics during pregnancy affects the production of cryptorchidism children. 
Sensibility analysis: Sensitivity analysis was used to check the stability of analysis results.

Country(ies) involved: China.

Keywords: cryptorchidism, nonsteroidal anti-inflammatory drugs.

Contributions of each author:

Author 1 - Wenliang Ge - Wenliang Ge independently extracted the data needed for single arm meta-analysis in the included study, including the author's name, study year, follow-up time, experimental type, number of cryptorchidism cases, number of cryptorchidism cases exposed to analgesics, type of analgesics and outcome measurement method.

Author 2 - Shiqi Ren - Shiqi Ren independently extracted the data needed for single arm meta-analysis in the included study, including the author's name, study year, follow-up time, experimental type, number of cryptorchidism cases, number of cryptorchidism cases exposed to analgesics, type of analgesics and outcome measurement method.

Author 3 - Yunzhao Xu - The difference between the two authors was referred to the third reviewer (Yunzhao Xu). 